retention rate (58\% in 2019 vs $100 \%$ in 2021 ) and decreased attrition rate ( $42 \%$ in 2019 vs $0 \%$ in 2021 ).

Conclusion. The Hertfordshire Community Perinatal Team has responded to the pandemic by innovating existing groups and creating new forums; many of which will continue on even after the pandemic ceases. The groups have acted as a lifeline for women breaking up the monotony and isolation of lockdown life and providing an invaluable space for women to be heard.

\section{Waiting list eradication in serious mental illness (SMI) "secondary care" psychology: addressing an NHS blind spot}

Nicola Airey ${ }^{1 \star}$, Zaffer Iqbal $^{2}$ and Sophie Brown ${ }^{3}$

${ }^{1}$ University of Manchester, Greater Manchester Mental Health NHS Foundation Trust; ${ }^{2}$ Navigo Heath and Social Care CiC, University of Hull and ${ }^{3}$ Navigo Health and Social Care CiC, University of Hull ${ }^{*}$ Corresponding author.

doi: 10.1192/bjo.2021.809

Aims. The poster focuses on the reduction, and eventual eradication, of waiting times within a community-based NHS psychology service in the North East of England. The poster aims to demonstrate the effectiveness of strategies implemented within a secondary care psychology service whilst examining patterns of help-seeking behaviour and treatment compliance in those waiting for therapy, and also the care needs of this cohort following a wait for services.

Background. Secondary care waiting lists for psychological therapy, as highlighted by a recent British Medical Association audit, remain a so-called 'blind-spot' in mental health care provision and a national problem. Tackling waiting lists within this sector has been stated as a priority within the Five Year Forward View, however "core ingredients" of waiting list eradication methodologies and the components leading to such, have yet to be disseminated.

Method. A historical audit and follow-up of clinical data were utilised to gather and analyse data of 208 individuals who were seen by the psychology service between October 2014 and March 2016. Result. No significant differences were found between individuals who successfully completed therapy compared to those who disengaged in regard to demographic or epidemiological variables, or mental health service input. Despite lengthy waiting times of up to 3.69 years, waiting time did not significantly impact whether someone engaged with psychological services. Any form of input from psychological services led to a significant reduction in distress, as measured by the CORE-OM. No individuals who completed therapy were re-referred for psychological input at 12-month follow-up. Conclusion. If imposed appropriately over a suitable time-frame evidence-based, effective and efficient needs-led psychological input can be provided whilst eradicating a waiting list and still remaining flexible, formulation-based and person-centred.

\section{Polypharmacy and potentially inappropriate} medications (PIMs) in older adults referred to a memory clinic

Anietie Akpan*, Bruno De Blaquiere, Issadevi Nellaya, Cornelia Termure and Sujoy Mukherjee

West London NHS Trust

${ }^{\star}$ Corresponding author.

doi: 10.1192/bjo.2021.810

Aims. The older adult is more likely to be prescribed a lot of medications (polypharmacy) on account of multi-morbidity and consequently being under the care of several specialists. Adverse drug events and reactions account for significant morbidity and mortality in this population group. Common sequelae include confusional episodes, dementia syndromes, falls, and higher rates of acute hospital admissions.Medications are not routinely reviewed in elderly care. We sought to estimate the prevalence of polypharmacy, and potentially inappropriate medications (e.g. anticholinergics or medications with central anticholinergic effects) in those referred to the Cognitive Impairment and Dementia Service (Elm Lodge), Older Persons Mental Health, West London NHS Trust.

Method. All referrals between 01/10/2020 and 30/11/2020 were screened for medications prescribed. Polypharmacy was defined as prescription of 5 or more medications. Medications with anticholinergic properties were considered examples of Potentially Inappropriate Medications (PIMs). The Anticholinergic Effect on Cognition (AEC) Tool, 'Medichec', was used to identify and rate anticholinergic burden. Anticholinergic load was also compared using the Anticholinergic Burden Scale (ABS).

Result. Total number of patients referred - 193

11 patients excluded due to unavailable/incomplete medication records.

Study number: 182

Polypharmacy:

$79.67 \%(\mathrm{n}=145)$ were prescribed 5 or more medications.

$44.51 \%(\mathrm{n}=81)$ prescribed 5-9 medications.

$23.08 \%(n=42)$ prescribed $10-14$ medications.

$8.79 \%(\mathrm{n}=16)$ prescribed $15-19$ medications.

$1.67 \%(\mathrm{n}=3)$ prescribed more than 20 medications.

Anticholinergics prescribed (AEC Tool):

$37.36 \%(\mathrm{n}=68)$ prescribed an anticholinergic.

$6.59 \%(n=12)$ prescribed more than 1 anticholinergic.

Anticholinergics (ABS):

$29.67 \%(\mathrm{n}=54)$ prescribed an anticholinergic.

$7.699 \%(\mathrm{n}=14)$ prescribed more than 1 anticholinergic.

Conclusion. Polypharmacy and potentially inappropriate prescribing (e.g. anticholinergics) remain widespread within the older adult population. Anticholinergic load was broadly similar with the Anticholinergic Effect on Cognition tool and the Anticholinergic Burden Scale. Increased anticholinergic burden further compounds risks of cognitive impairment, delirium and death.Other categories of Potentially Inappropriate Medications, including those no longer needed, ought to be identified and reviewed. Over-the-counter medications also need to be screened for.

Elimination or reduction of anticholinergic burden may improve quality of life for patients, as well as cost burden on services.

Pharmacovigilance, collaborative working, and regular training are needed across services providing care for the older adult.

Polypharmacy and potentially inappropriate medications (PIMS) in older adults referred to a liaison psychiatry service

Anietie Akpan* and Omolade Longe

West London NHS Trust

${ }^{*}$ Corresponding author.

doi: 10.1192/bjo.2021.811 